\title{
BINARY STARS OBSERVED WITH ADAPTIVE OPTICS AT THE STARFIRE OPTICAL RANGE
}

\author{
JACK D. DRUMMOND \\ Air Force Research Laboratory, Directed Energy Directorate, RDSAM, 3550 Aberdeen Avenue SE, Kirtland AFB, NM 87117-5776, USA \\ Received 2013 November 30; accepted 2013 December 20; published 2014 February 11
}

\begin{abstract}
In reviewing observations taken of binary stars used as calibration objects for non-astronomical purposes with adaptive optics on the $3.5 \mathrm{~m}$ Starfire Optical Range telescope over the past 2 years, one-fifth of them were found to be off-orbit. In order to understand such a high number of discrepant position angles and separations, all previous observations in the Washington Double Star Catalog for these rogue binaries were obtained from the Naval Observatory. Adding our observations to these yields new orbits for all, resolving the discrepancies. We have detected both components of $\gamma$ Gem for the first time, and we have shown that 7 Cam is an optical pair, not physically bound.
\end{abstract}

Key words: binaries: close - binaries: general - binaries: visual - instrumentation: adaptive optics

Online-only material: color figures

\section{INTRODUCTION AND BACKGROUND}

The Starfire Optical Range (SOR), a facility owned and operated by the Directed Energy Directorate of the Air Force Research Laboratory on Kirtland Air Force Base near Albuquerque, New Mexico, currently uses its $3.5 \mathrm{~m}$ telescope for research and development into atmospheric compensation using adaptive optics (AO). For the most recent description of the AO system, see Johnson et al. (2009). During the course of non-astronomical experiments, binary stars are often observed for calibration and orientation purposes. To this end, a list of calibration binaries was hand-selected by the author from fixed binaries and binaries with good orbits. Subsequently, another list was compiled from the Sixth Catalog of Orbits of Visual Binaries from the Washington Double Star (WDS) Catalog (http://ad.usno.navy.mil/wds) maintained by the United States Naval Observatory, and both were made available as Excel spreadsheets at the annual AMOS Conference (Drummond 2011, 2012). These spreadsheets automatically calculate position angles (P.A.s) and separations for a given time.

Observations of these binaries with $\mathrm{AO}$, in Natural Guide Star mode (not using our sodium guide star laser) on the $3.5 \mathrm{~m}$ telescope, showed that many were off from their predicted positions. Over 23 nights between 2010 June 18 and 2012 April 6, during the course of many other experiments, 174 observations of 62 binaries were recorded from these two lists. All observations were made at an effective broadband wavelength of $0.78 \mu \mathrm{m}$, or through a narrowband $\mathrm{H} \alpha$ filter at $0.66 \mu \mathrm{m}$ with the same Andor camera. Each binary was measured by fitting the pair as Lorentzians, since this function best describes the AO point-spread function (Drummond 1998; Drummond et al. 1998), and for close pairs (most of them), both components were forced to have the same shape in the fit, the isoplanatic assumption.

After comparing measurements to the predictions from the two lists, Figure 1 shows the scale obtained from 144 measurements of 50 binaries, and a histogram of the differences between the observed and predicted P.A.s for these same binaries. Figure 2 shows the latter converted to an arc distance. Not shown in either figure are 12 of the 62 binaries that were deemed to be less than calibration material because they are "off-orbit," where the criteria for exclusion were a $0{ }^{\prime} 1$ or $10 \%$ discrepancy in predicted separation.

\section{RESOLUTION}

Orbits used to calculate the position and orientation of components are the orbits available at the time of the observation. In an attempt to understand so many discrepancies among socalled calibration binaries, the Naval Observatory was queried for their database of observations for the 12 cases of off-orbit binaries. Since the end of the last SOR observation here in 2012 April, new observations and orbits have appeared in the WDS catalog for some of the discrepant pairs, and in each case, by adding observations (including ours) unavailable to the last orbit calculator, we find a new orbit that dissolves the apparent discrepancy.

In most cases, modern $\mathrm{CCD}$, speckle interferometry, or especially AO observations provide the latest and best data points, and are given a weight of 20 in calculating an orbit as compared to a weight of unity for visual observations, in accordance with previous AO work, e.g., Mason et al. (1995). Table 1 gives the J2000 date, P.A.s, and separations obtained at the SOR for these 12 discrepant binaries (counting WDS $08592+4803$ as two), Table 2 gives the old and new orbits, and Figures $3-15$ show old and new orbits calculated here after including all data, along with some images.

In all of the orbit figures, points, which are the B components, are connected to their predicted positions on the orbit. Open circles are from visual measurements and large filled circles are CCD, speckle interferometry, or AO measurements. Points marked as an $\mathrm{X}$ or connected to the orbit with a dotted line were not available or not used in the orbit calculation. The plus marks the A component and the dashed line through it is the line of apsides, while the dash-dotted line is the line of nodes with an arrow showing the direction of motion just off the ascending node. Times along the orbit are shown as smaller dots. Units are seconds of arc.

\section{INDIVIDUAL SYSTEMS}

$$
\begin{aligned}
& \text { 3.1. WDS 00318+5431=STT } 12= \\
& \text { HIP } 2505=H R 123=14 \lambda \text { Cas }
\end{aligned}
$$

One spectroscopic determination of position and separation, even after changing P.A. by $180^{\circ}$, is not used in the fit. The new orbit has a shorter period, is less eccentric, and is smaller. 

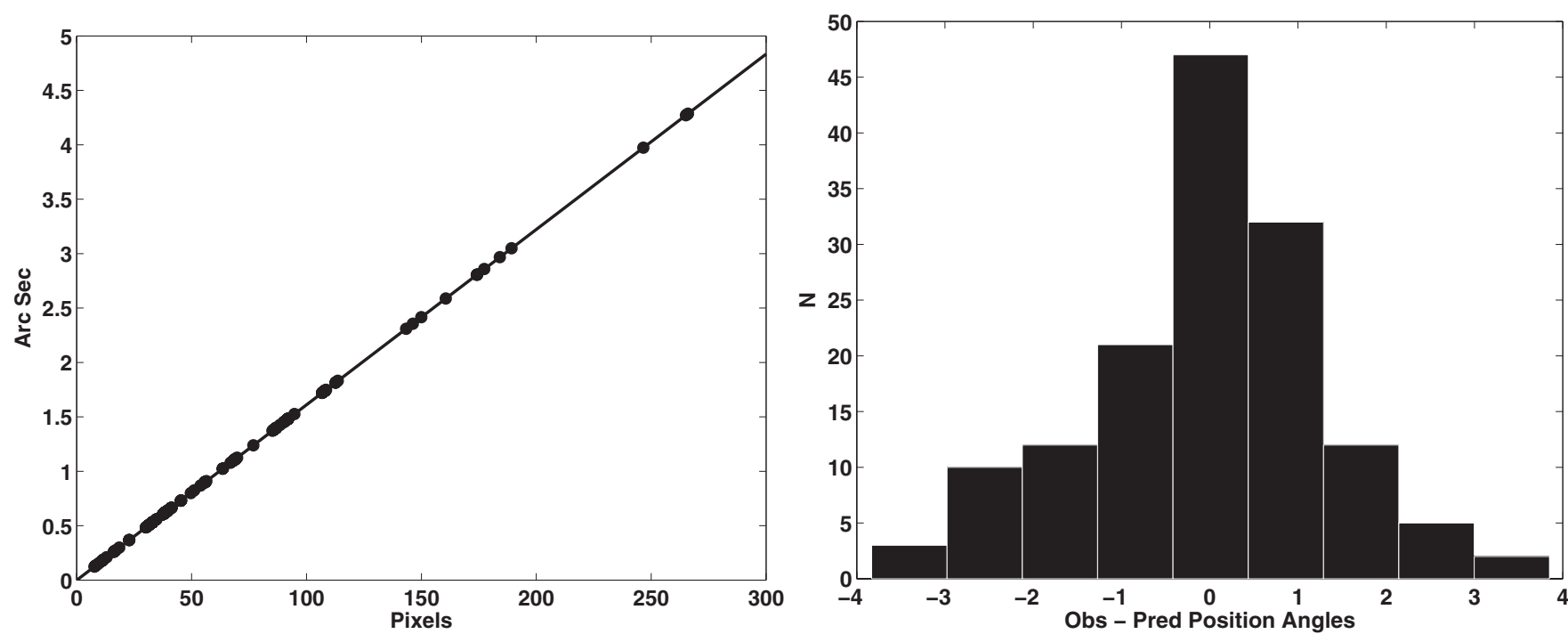

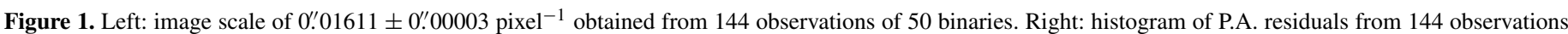
of 62 binaries. The standard deviation from a Gaussian fit is $\sigma=1.0$.

Table 1

SOR Observations of Discordant Binaries

\begin{tabular}{|c|c|c|c|c|c|c|c|}
\hline WDS & Date & $\begin{array}{l}\text { P.A. } \\
\left({ }^{\circ}\right)\end{array}$ & $\begin{array}{c}\text { Sep } \\
\left({ }^{\prime \prime}\right)\end{array}$ & $\Delta$ Mag & Filter $^{\mathrm{a}}$ & Obs & Nights \\
\hline $00318+5431$ & 2010.654 & $209.8 \pm 0.1$ & $0.233 \pm 0.001$ & $0.07 \pm 0.01$ & $R$ & 1 & 1 \\
\hline \multirow[t]{2}{*}{$04573+5345$} & 2010.652 & $203.2 \pm 0.2$ & $0.560 \pm 0.018$ & $3.15 \pm 0.03$ & $R$ & 1 & 1 \\
\hline & 2011.774 & $202.3 \pm 0.2$ & $0.561 \pm 0.017$ & $3.12 \pm 0.04$ & $\mathrm{H} \alpha$ & 1 & 1 \\
\hline \multirow[t]{2}{*}{$05413+1632$} & 2010.913 & $244.4 \pm 0.3$ & $0.210 \pm 0.002$ & $1.52 \pm 0.02$ & $R$ & 1 & 1 \\
\hline & 2011.145 & $248.5 \pm 0.3$ & $0.216 \pm 0.001$ & $1.79 ? \pm 0.01$ & $R$ & 1 & 1 \\
\hline $06377+1624$ & 2012.259 & $259.3 \pm 0.3$ & $0.378 \pm 0.002$ & $3.06 \pm 0.03$ & $\mathrm{H} \alpha$ & 1 & 1 \\
\hline \multirow[t]{2}{*}{$08468+0625 \mathrm{AC}$} & 2010.912 & $303.3 \pm 0.1$ & $2.779 \pm 0.012$ & $3.76 \pm 0.10$ & $R$ & 1 & 1 \\
\hline & 2012.259 & $303.9 \pm 0.1$ & $2.745 \pm 0.001$ & $4.51 \pm 0.02$ & $\mathrm{H} \alpha$ & 1 & 1 \\
\hline $08592+4803 \mathrm{AB}$ & 2012.260 & $95.9 \pm 0.4$ & $1.925 \pm 0.010$ & $6.93 \pm 0.27$ & $\mathrm{H} \alpha$ & 2 & 2 \\
\hline $\mathrm{AC}$ & 2012.260 & $81.5 \pm 0.3$ & $2.398 \pm 0.010$ & $6.97 \pm 0.29$ & $\mathrm{H} \alpha$ & 2 & 2 \\
\hline $\mathrm{BC}$ & 2012.260 & $39.6 \pm 0.3$ & $0.716 \pm 0.001$ & $0.04 \pm 0.01$ & $\mathrm{H} \alpha$ & 2 & 2 \\
\hline $10281+4847$ & 2012.260 & $22.0 \pm 0.1$ & $3.975 \pm 0.015$ & $4.31 \pm 0.04$ & $\mathrm{H} \alpha$ & 4 & 3 \\
\hline $12533+2115$ & 2011.223 & $193.2 \pm 0.1$ & $1.135 \pm 0.002$ & $2.85 \pm 0.10$ & $R$ & 3 & 2 \\
\hline $13007+5622$ & 2012.262 & $108.0 \pm 0.1$ & $0.992 \pm 0.001$ & $2.61 \pm 0.04$ & $\mathrm{H} \alpha$ & 2 & 2 \\
\hline $13473+1727$ & 2012.257 & $56.7 \pm 0.1$ & $1.829 \pm 0.008$ & $5.72 \pm 0.18$ & $\mathrm{H} \alpha$ & 2 & 2 \\
\hline \multirow[t]{4}{*}{$15496-0326$} & 2011.216 & $342.2 \pm 0.2$ & $0.233 \pm 0.002$ & $1.62 \pm 0.05$ & $R$ & 2 & 1 \\
\hline & 2011.225 & $343.5 \pm 0.1$ & $0.234 \pm 0.001$ & $1.77 \pm 0.01$ & $R$ & 1 & 1 \\
\hline & 2011.320 & $340.9 \pm 0.1$ & $0.236 \pm 0.001$ & $1.91 \pm 0.01$ & $R$ & 1 & 1 \\
\hline & 2011.474 & $341.4 \pm 0.3$ & $0.241 \pm 0.001$ & $1.87 \pm 0.02$ & $R$ & 1 & 1 \\
\hline
\end{tabular}

Note. ${ }^{\mathrm{a}} \lambda_{R}=0.78 \mu \mathrm{m} ; \Delta \lambda_{R}=0.10 \mu \mathrm{m} ; \lambda_{\mathrm{H} \alpha}=0.656 \mu \mathrm{m} ; \Delta \lambda_{\mathrm{H} \alpha}=0.004 \mu \mathrm{m}$.

$$
\begin{aligned}
& \text { 3.2. WDS } 04573+5345=D 5= \\
& H I P 23040=H R 1568=7 \text { Cam }
\end{aligned}
$$

Pre-1979 observations listed the fainter companion as A, but afterward, more appropriately as $\mathrm{B}$, which then makes the longitude of the ascending node differ by $180^{\circ}$ from that of Baize (1979). Straightening out the ambiguities clearly shows that the two stars are not physically related, but are an optical pair. A more recent long period orbit $(2733 \mathrm{yr})$ has been derived by Romero (2013) that leads to small differences between observed and predicted positions. However, using this as a starting orbit, we are unable to confirm (converge to) this orbit.

$$
\begin{aligned}
& \text { 3.3. WDS } 05413+1632=B U 1007= \\
& \text { HIP } 26777=H R 1946=126 \text { Tau }
\end{aligned}
$$

There is not much of a difference in appearance between the previous and new orbits. One AO point from 2001.0985 is not used. Old visual positions produce lots of scatter and, likewise, our two points are part of the surprising scatter of AO and SI observations. The database magnitude differences imply that one or both stars may be variable in brightness.

\subsection{WDS $06377+1624 A a A b=O C C 9011=$ $H I P 31681=H R 2421=\gamma$ Gem}

The previous orbit derived from Hipparcos observations (Jancart et al. 2005) of the perturbations on the parallactic ellipse refers to the motion of component A around the center of mass. With our detection of both components we can set the scale of the astrometric/spectroscopic orbital elements, and with the parallax from Hipparcos (van Leeuwen 2007) of $0^{\prime \prime} .02984 \pm 0.00223$, we derive the mass of the two as $3.4 \pm 0.8 M_{\odot}$ and $1.4 \pm 0.3 M_{\odot}$. 
Table 2

Previous and New Orbits

\begin{tabular}{|c|c|c|c|c|c|c|c|c|}
\hline WDS & $\begin{array}{c}a \\
\left({ }^{\prime \prime}\right)\end{array}$ & $\begin{array}{l}\text { Node } \\
\left({ }^{\circ}\right)\end{array}$ & $\begin{array}{c}i \\
\left({ }^{\circ}\right)\end{array}$ & $\begin{array}{l}\text { Per } \\
(\mathrm{yr})\end{array}$ & $T_{0}$ & $e$ & $\begin{array}{l}\omega \\
\left({ }^{\circ}\right)\end{array}$ & Reference \\
\hline \multicolumn{9}{|l|}{$00318+5431$} \\
\hline Prev & 1.165 & 17.4 & 75.8 & 536.47 & 2012.09 & 0.816 & 260.5 & Ling et al. 2005 \\
\hline $\begin{array}{l}\text { New } \\
04573+5345\end{array}$ & $0.448 \pm 0.028$ & $17.6 \pm 9.6$ & $53.6 \pm 5.2$ & $246.54 \pm 36.96$ & $2012.54 \pm 4.65$ & $0.689 \pm 0.119$ & $301.0 \pm 2.6$ & this paper \\
\hline $\begin{array}{l}\text { Prev } \\
\text { New }^{a}\end{array}$ & 0.78 & 158.2 & 137.9 & 284.0 & 1986.0 & 0.74 & 30.2 & Baize 1979 \\
\hline $05413+1632$ & & & & & & & & \\
\hline Prev & $0.316 \pm 0.133$ & $48.4 \pm 3.0$ & $81.8 \pm 4.0$ & $114.88 \pm 2.00$ & $1941.25 \pm 6.00$ & $0.869 \pm 0.092$ & $61.1 \pm 16.0$ & Docobo \& Ling 1999 \\
\hline $\begin{array}{l}\text { New } \\
06377+1624\end{array}$ & $0.245 \pm 0.014$ & $53.2 \pm 0.8$ & $80.5 \pm 1.1$ & $111.02 \pm 1.37$ & $1938.13 \pm 1.64$ & $0.661 \pm 0.036$ & $40.9 \pm 5.8$ & This paper \\
\hline $\begin{array}{l}\text { A astrometric } \\
\text { AB relative } \\
08468+0625\end{array}$ & $\begin{array}{c}0.0787 \pm 0.0023 \\
0.2732\end{array}$ & $243.6 \pm 2.6$ & $106.7 \pm 1.7$ & 12.634 & 1979.34 & 0.89 & $\begin{array}{l}312.6 \\
132.6\end{array}$ & $\begin{array}{l}\text { Jancart et al. } 2005 \\
\text { This paper }\end{array}$ \\
\hline Prev & 4.66 & 49.3 & 39 & 990 & 1920 & 0.30 & 200 & Heintz 1996 \\
\hline $\begin{array}{l}\text { New } \\
08592+4803\end{array}$ & $3.37 \pm 0.01$ & $34.5 \pm 1.7$ & $33.2 \pm 0.4$ & $589 \pm 5$ & $2159 \pm 2$ & 0 & 0 & This paper \\
\hline A-BC Prev & 9.092 & 184.8 & 57.8 & 817.91 & 2402.86 & 0.79 & 129.7 & Hopmann 1973 \\
\hline New & $11.3 \pm 0.2$ & $169.0 \pm 0.5$ & $78.9 \pm 0.3$ & $803 \pm 25$ & $2209.6 \pm 6.3$ & 0 & 0 & This paper \\
\hline BC Prev & $0.68 \pm 0.01$ & 21.0 & $108 \pm 1$ & $39.69 \pm 0.53$ & $1918.58 \pm 0.66$ & $0.32 \pm 0.02$ & 338.3 & Eggen 1967 \\
\hline $\begin{array}{l}\text { New } \\
10281+4847\end{array}$ & $0.70 \pm 0.02$ & $21.5 \pm 2.1$ & $110.9 \pm 1.1$ & $38.82 \pm 0.11$ & $1919.49 \pm 0.45$ & $0.32 \pm 0.01$ & $9.9 \pm 3.7$ & This paper \\
\hline Prev & 7.08 & 18.94 & 72.01 & 765 & 1997 & 0.53 & 67.74 & Hale 1994 \\
\hline $\begin{array}{l}\text { New } \\
12533+2115\end{array}$ & $4.67 \pm 0.12$ & $16.5 \pm 1.9$ & $81.4 \pm 3.5$ & $590 \pm 208$ & $1958.6 \pm 16.8$ & 0 & 0 & This paper \\
\hline Prev & 1.181 & 201.4 & 34 & 359 & 2038.0 & 0.145 & 30 & Heintz 1997 \\
\hline $\begin{array}{l}\text { New } \\
13007+5622\end{array}$ & $1.405 \pm 0.046$ & $238.7 \pm 2.7$ & $28.4 \pm 13.4$ & $539.4 \pm 95.4$ & $1949.4 \pm 7.9$ & $0.208 \pm 0.100$ & $251.6 \pm 7.3$ & This paper \\
\hline Prev & $1.250 \pm 0.042$ & $95.4 \pm 1.5$ & $50.4 \pm 1.4$ & $106.4 \pm 0.85$ & $1921.828 \pm 0.44$ & $0.412 \pm 0.013$ & $113.9 \pm 1.1$ & Scardia et al. 2005 \\
\hline $\begin{array}{l}\text { New } \\
13473+1727\end{array}$ & $1.208 \pm 0.010$ & $88.0 \pm 1.1$ & $46.9 \pm 0.9$ & $104.9 \pm 0.6$ & $1921.224 \pm 0.403$ & $0.388 \pm 0.009$ & $119.2 \pm 1.5$ & This paper \\
\hline Prev & 14.39 & 28.4 & 50.7 & 2000 & 2017 & 0.91 & 99.3 & Hale 1994 \\
\hline $\begin{array}{l}\text { New } \\
15496-0326\end{array}$ & $7.60 \pm 6.90$ & $171.9 \pm 50.3$ & $37.0 \pm 39.7$ & $964 \pm 1321$ & $2030 \pm 5$ & $0.836 \pm 0.074$ & $334.2 \pm 28.6$ & This paper \\
\hline Prev & 0.35 & $116 \pm 28$ & $103 \pm 28$ & $36 \pm 2$ & $1988.9 \pm 1.8$ & $0.4 \pm 0.3$ & $308 \pm 32$ & Gontcharov \& Kiyaeva 2010 \\
\hline New & $0.255 \pm 0.012$ & $223.0 \pm 2.4$ & $131.0 \pm 3.6$ & $35.86 \pm 1.91$ & $2003.96 \pm 0.27$ & $0.750 \pm 0.024$ & $97.9 \pm 1.5$ & This paper \\
\hline
\end{tabular}

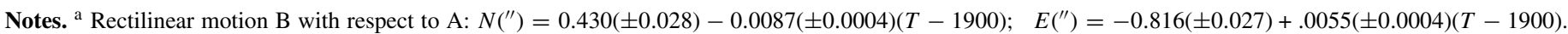
The closest approach occurred at 1977.5.

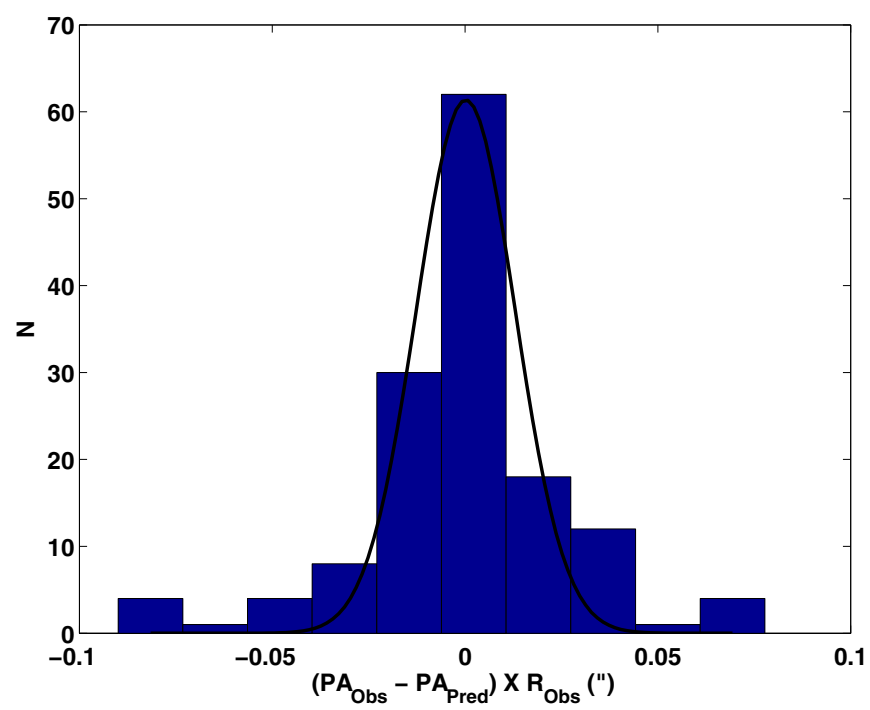

Figure 2. Histogram of the arc distance of P.A. residuals. Standard deviation from a Gaussian fit translates the P.A. $\sigma=1.0$ in Figure 1 to $0^{\prime \prime} 013$.

(A color version of this figure is available in the online journal.)

\subsection{WDS $08468+0625$ AC $=S T F 1273=$ $H I P 43109=H R 3482=11 \epsilon H y a$}

While an orbit with a non-zero eccentricity can be found of $0.079 \pm 0.20$, its uncertainty suggests that it is better to assume a circular orbit, which consequently reduces all of the uncertainties of the other orbital elements.

\section{6. $W D S 08592+4803 A-B C=H J 2477=$ $H I P 44127=H R 3569=9 \iota U M a$}

Apparently, B and C (Figure 8) were not recorded separately from A until 2008. Therefore, the averages of the two AO measurements in 2008 (De Rosa et al. 2011) are computed and used, as well as the two in 2010 (De Rosa et al. 2011), the two CCD measurements in 2009 (Rica et al. 2012), and our own in 2012. These last four observations are then given the higher $\mathrm{AO} / \mathrm{SI}$ weight of 20 compared to unity for all others. A non-zero eccentricity orbit fit could not be found, and therefore the orbit is fit as circular. One P.A. from 1903 was changed by $180^{\circ}$. 


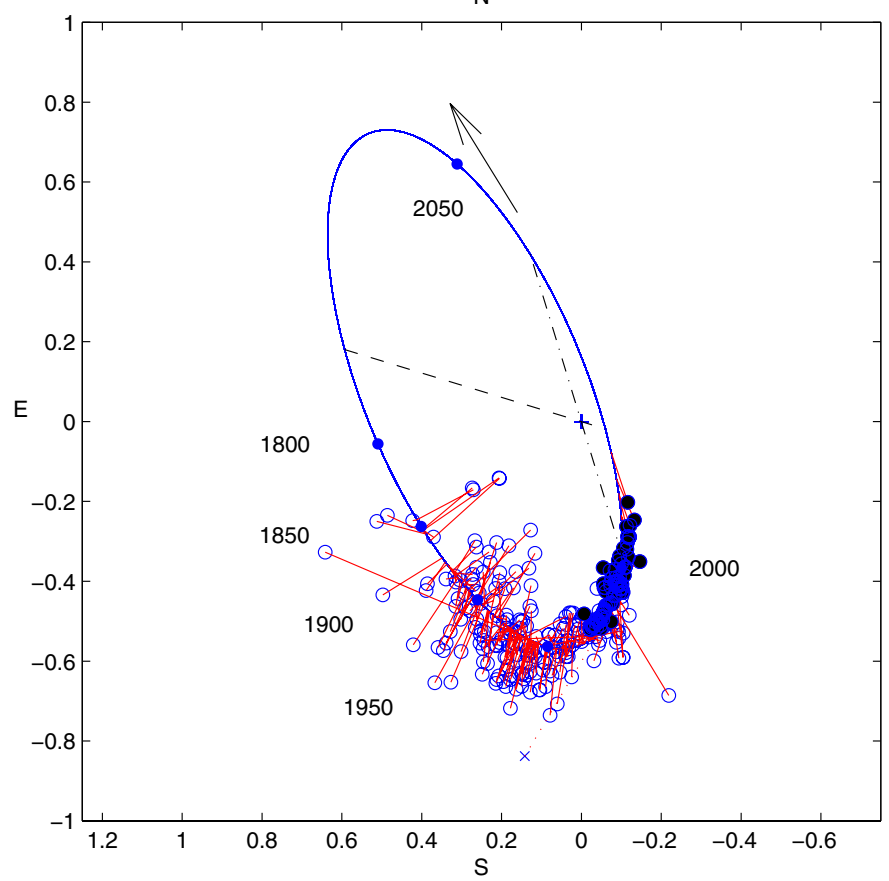

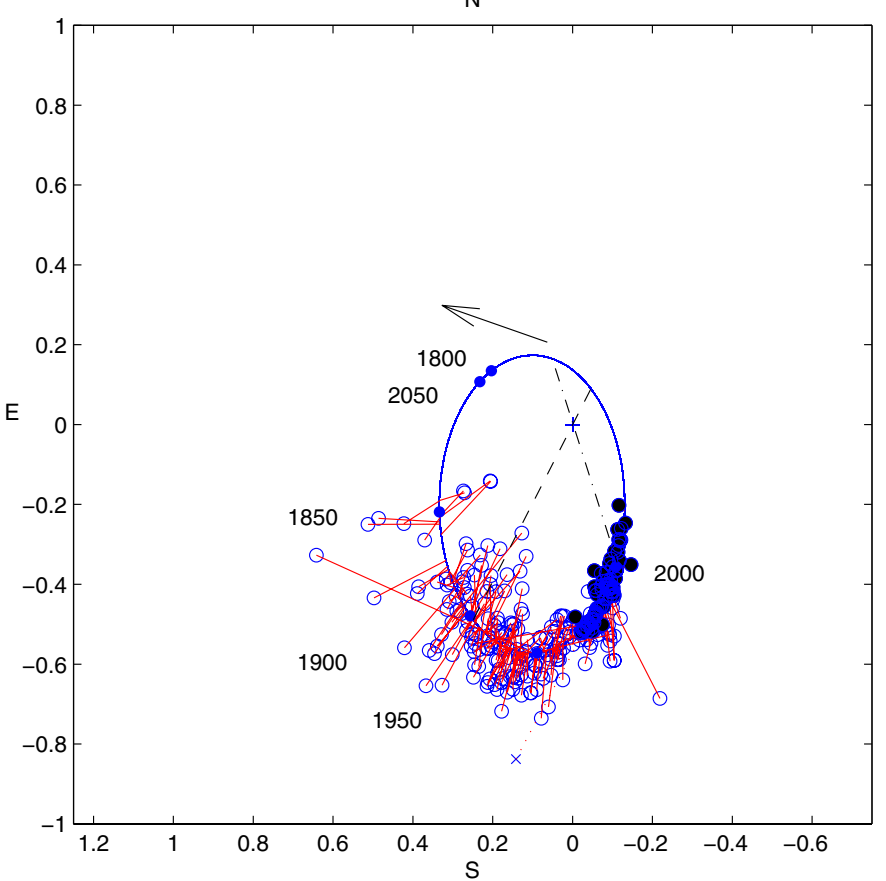

Figure 3. WDS 00318+5431. Left: previous orbit. Right: new orbit.

(A color version of this figure is available in the online journal.)
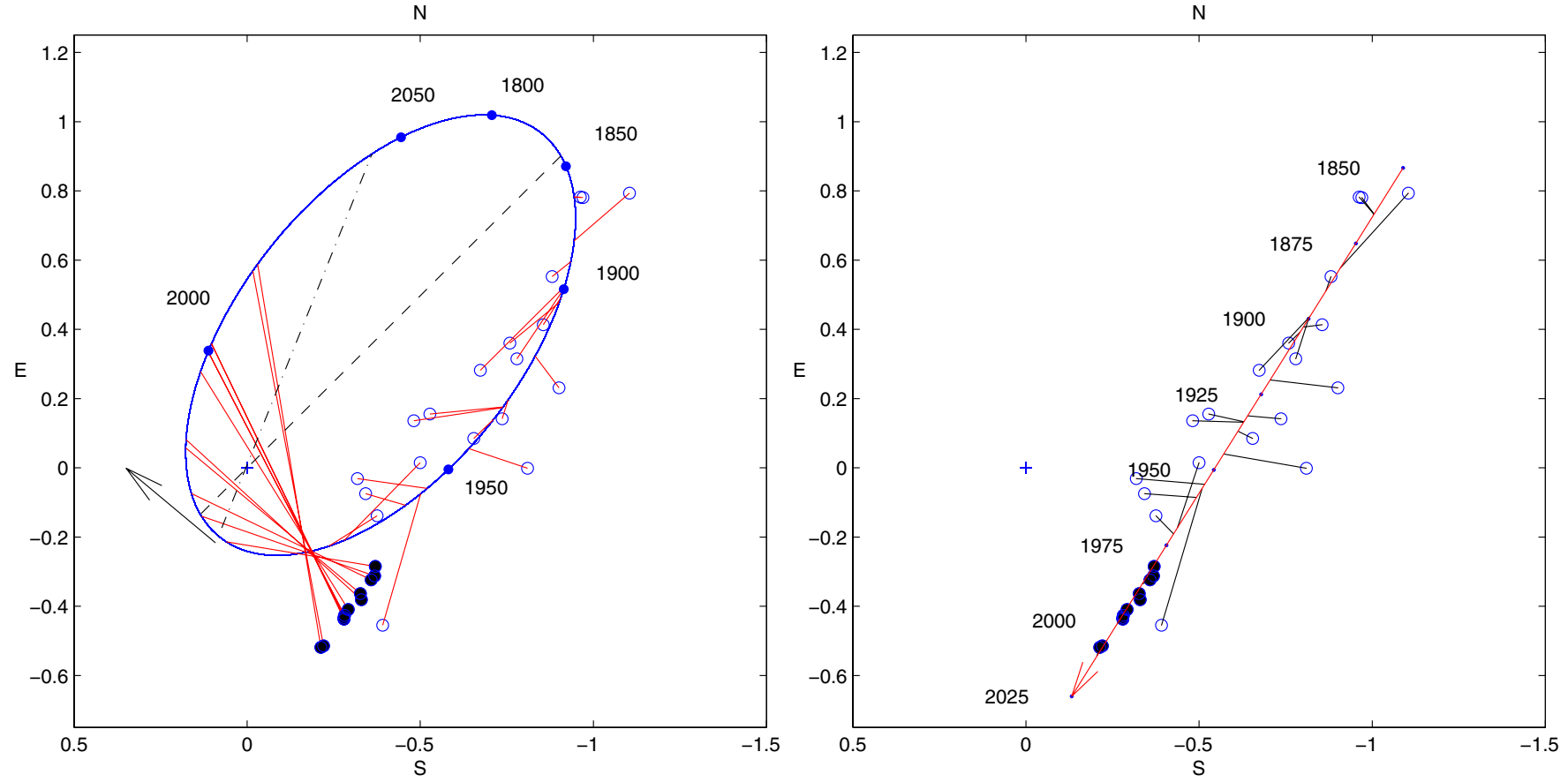

Figure 4. WDS 04573+5345. Left: previous orbit. Right: rectilinear motion of 7 Cam.

(A color version of this figure is available in the online journal.)

For $\iota \mathrm{UMa} \mathrm{BC}$ (HU 628), since the B and C components have nearly the same brightness which can lead to an ambiguous identification, and guided by the orbit of Eggen (1967), $180^{\circ}$ were added to nine P.A.s, and two other points were not used. There is not a significant difference between the previous and the new orbits, but there may be evidence for sub-orbital motion indicating a third component since modern $\mathrm{AO} / \mathrm{SI}$ measurements clearly show greater separation than observations from earlier in the last century.

\subsection{WDS $10281+4847=K U I 50=H I P 51248=H R 4098$}

The orbit of Hale (1994) includes his observation as the final one, but subsequently it is shown to be bad and is not used in 


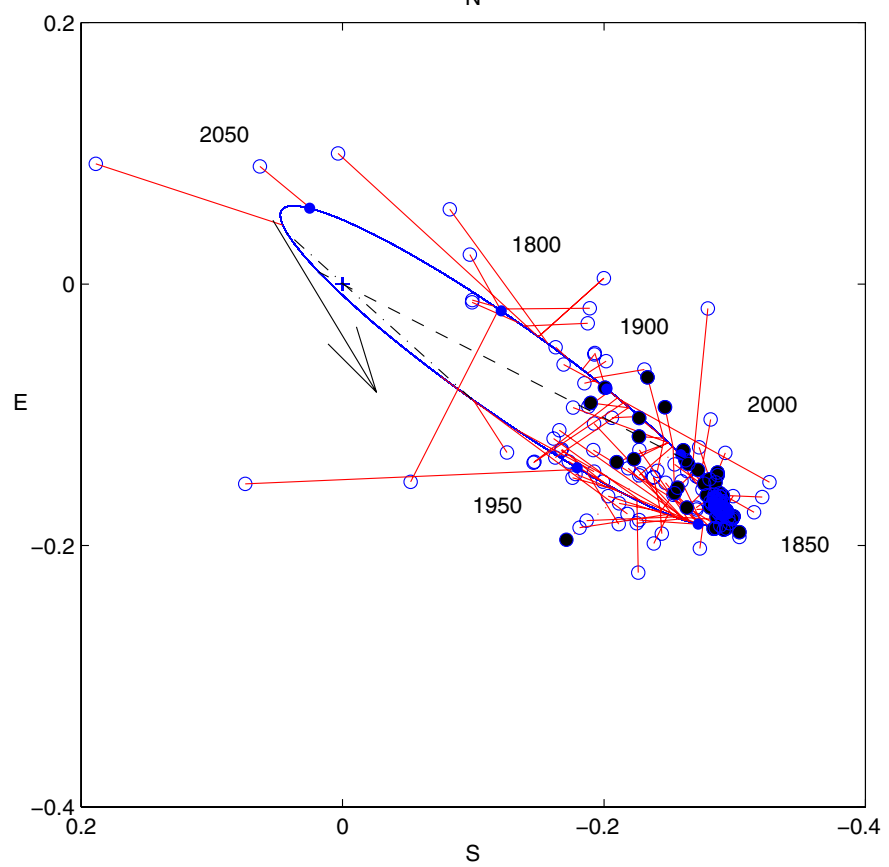

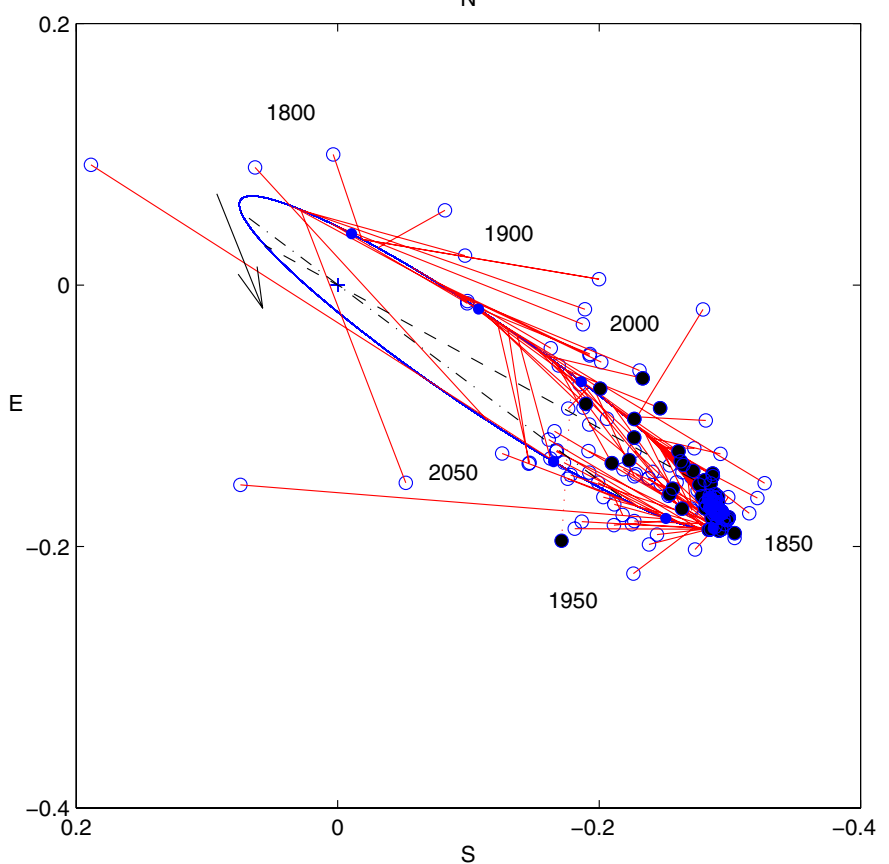

Figure 5. WDS 05413+1632. Left: previous orbit. Right: new orbit. Component B is now well past apastron. (A color version of this figure is available in the online journal.)
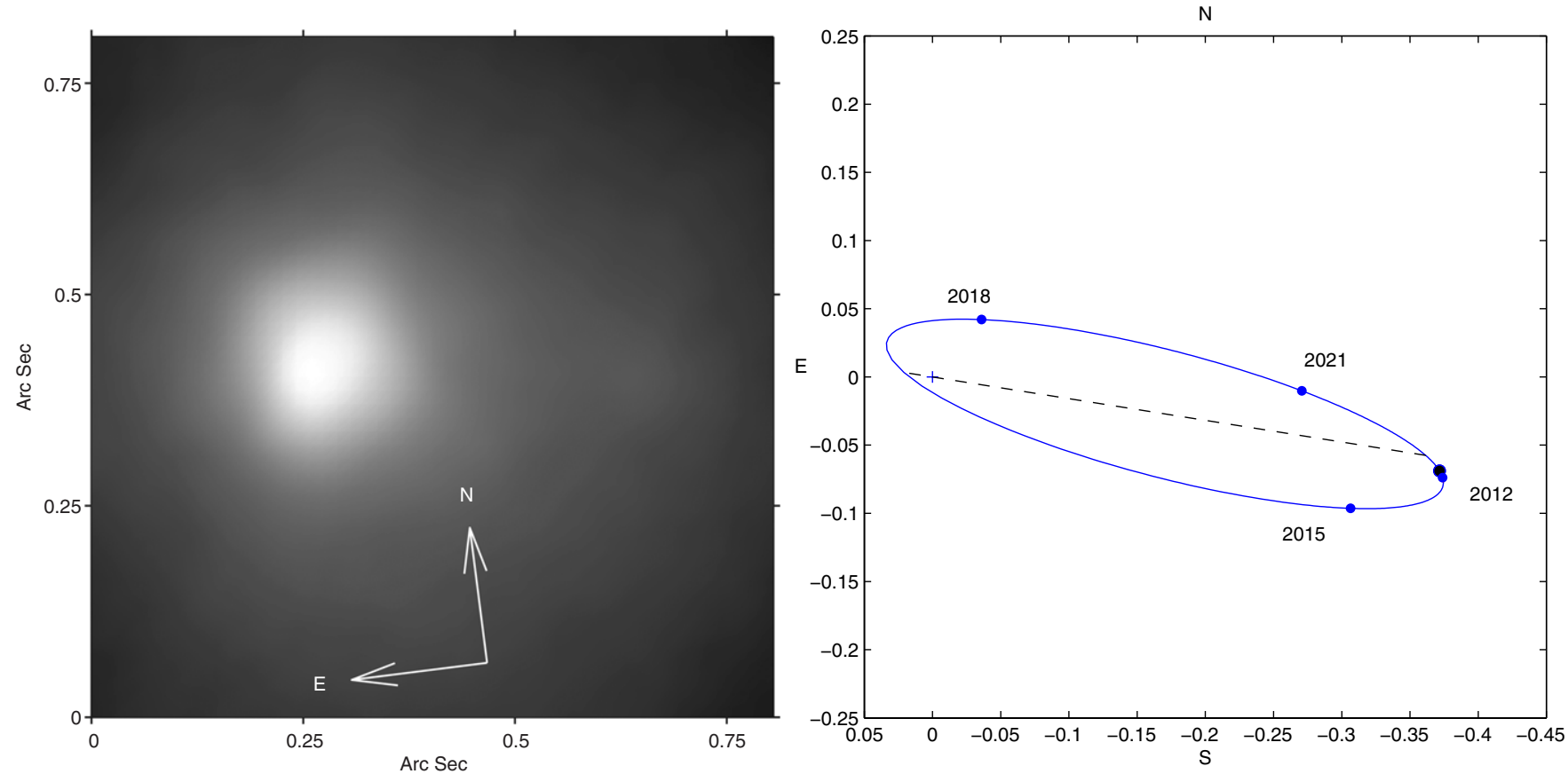

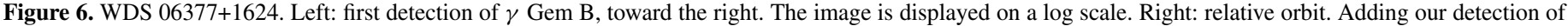
both components to the astrometric and spectroscopic orbital elements sets the scale for both.

(A color version of this figure is available in the online journal.) 


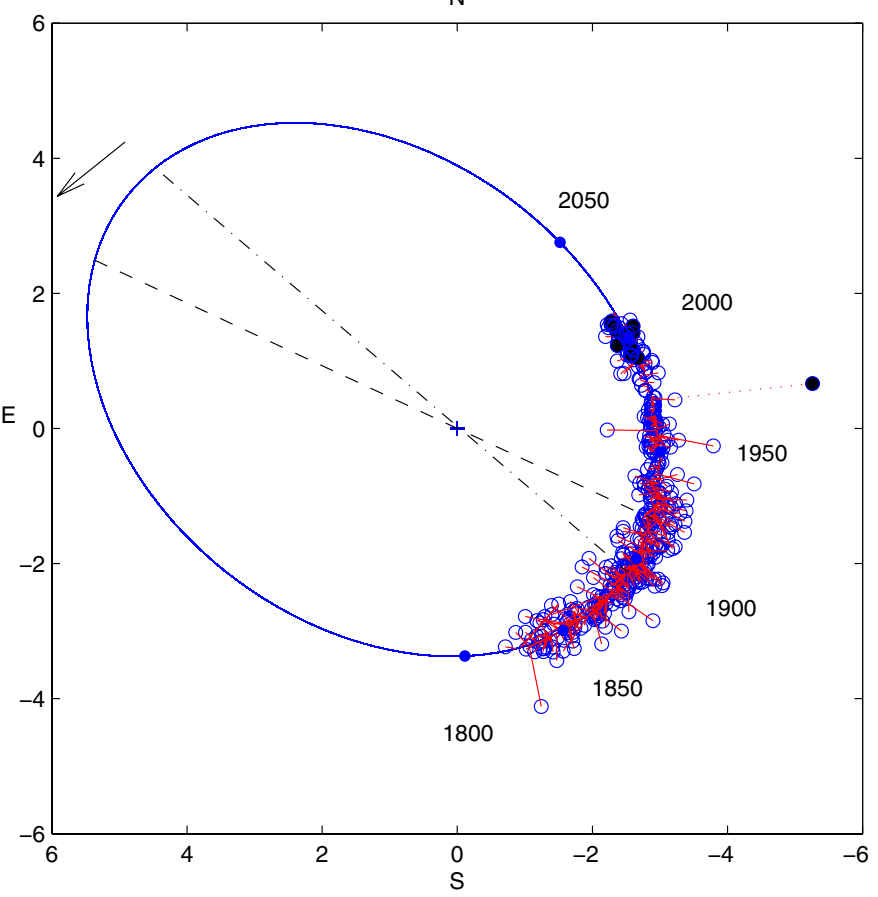

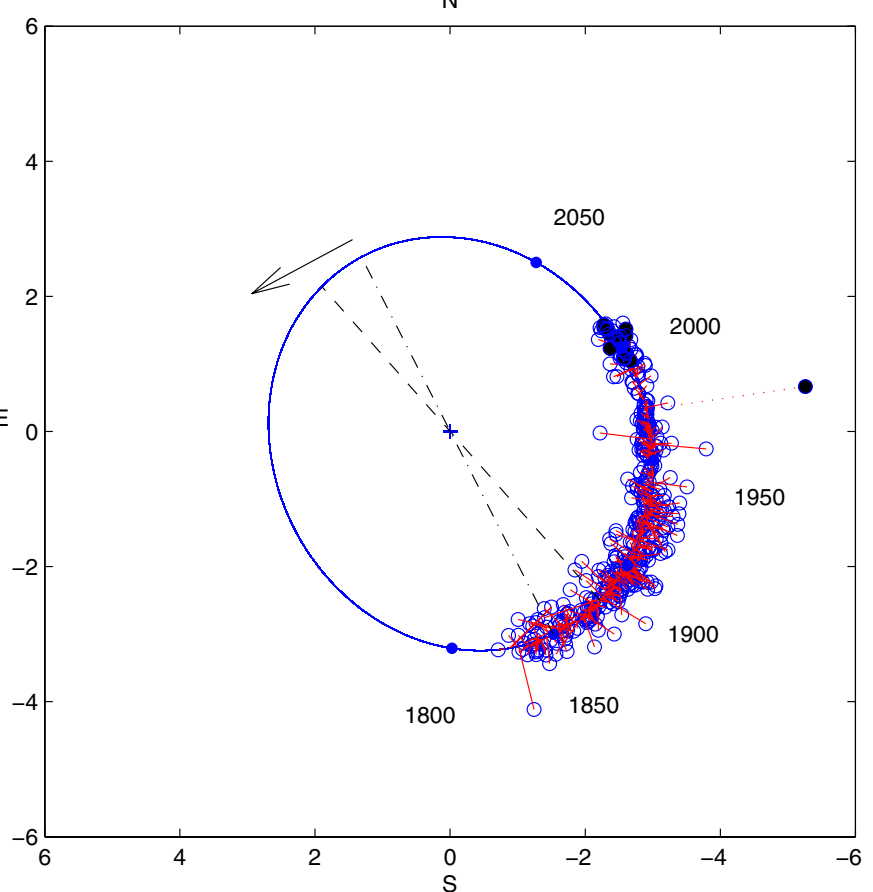

Figure 7. WDS $08468+0625$ AC. Left: previous orbit. Right: new orbit. (A color version of this figure is available in the online journal.)

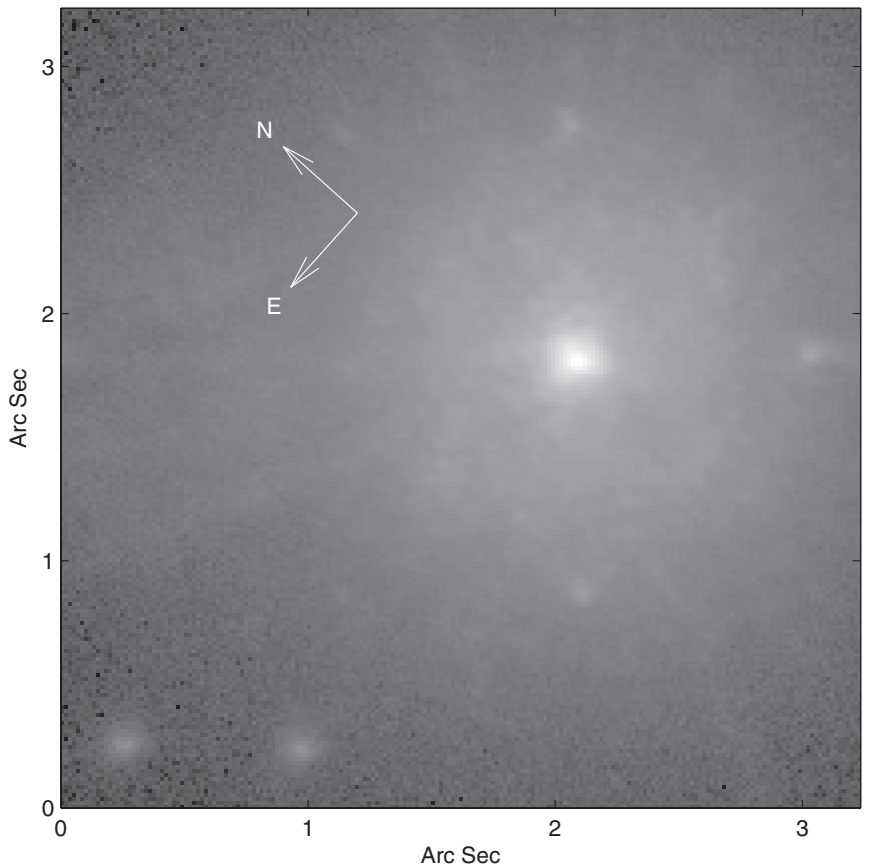

Figure 8. WDS $05892+4803$. At lower left, component $B$ is to the left of component $\mathrm{C}$. The four spots (one is very faint) around component A are familiar waffle spot artifacts of AO.

a new orbit calculation. Another newer point is the average of four simultaneous rather widely scattered measurements. Only our AO point is given a high weight. Clearly the orbit has a high inclination but no eccentricity could be found for this premature orbit.

\section{8. $W D S 12533+2115=S T F 1687=$ HIP $62886=H R 4894=35$ Com}

Recent AO, SI, and CCD measurements suggest a longer period with higher eccentricity. It also appears that the system is receding from, not approaching, periastron.

$$
\begin{aligned}
& \text { 3.9. WDS } 13007+5622=B U 1082= \\
& H I P 63503=H R 4931=78 U M a
\end{aligned}
$$

The new orbit calculated here is not much different from the previous one by Scardia et al. (2005), nor from an even more recent one by Scardia et al. (2012), but the difference between the predicted and our measured positions in 2012 is significant, greater than the threshold for being discrepant.

$$
\begin{aligned}
& \text { 3.10. WDS } 13473+1727=S T T 270+ \\
& \text { HIP } 67275=H R 5185=4 \text { ¿ Boo }
\end{aligned}
$$

The previous orbit by Hale (1994), a more recent one by Roberts et al. (2011), and ours, all have long periods and high eccentricities for this high contrast binary. The first three observations from the early 1800s were not used in the current fit.

$$
\begin{gathered}
\text { 3.11. WDS } 15496-0326=C H R 259=H I P 77516= \\
H R 5881=32 \mu \text { Ser }
\end{gathered}
$$

In order to keep the fainter companion orbiting the brighter component, $180^{\circ}$ are added to all of the reported P.A.s, as well as to the node of the previous orbit by Gontcharov \& Kiyaeva (2010), which is clearly incorrect. The new orbit fits the recent $\mathrm{AO}$ and SI measurements much better, and an even more recent orbit by Cvetkovic (2011) is nearly the same as ours here. The Hipparcos measurement is not used. 


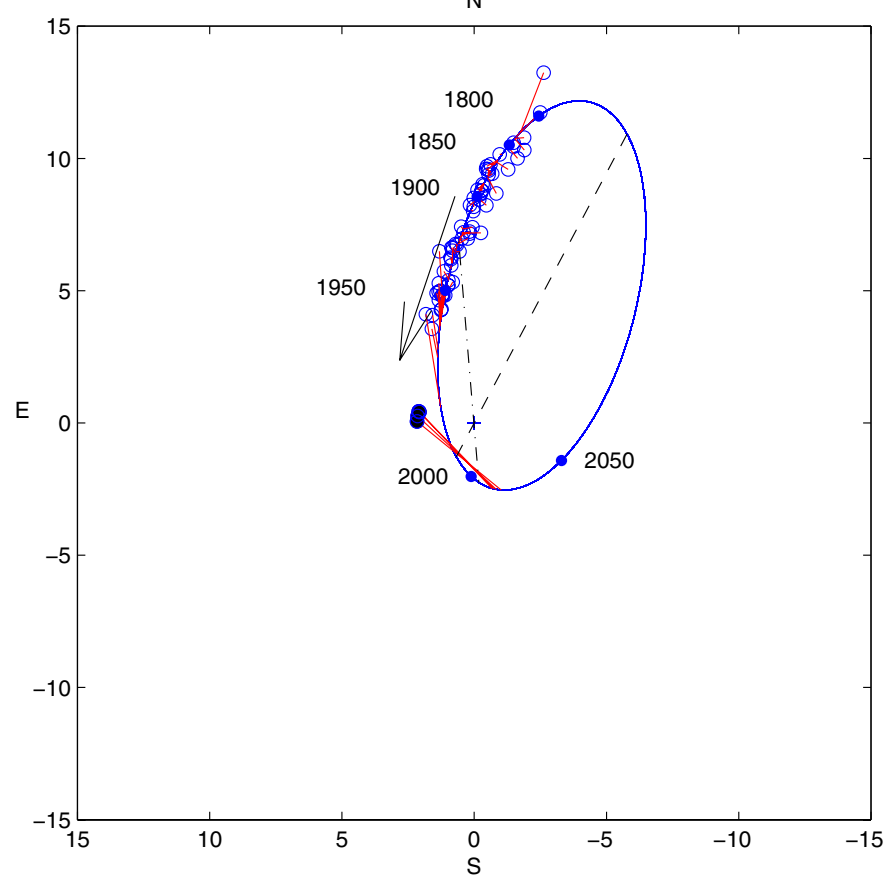

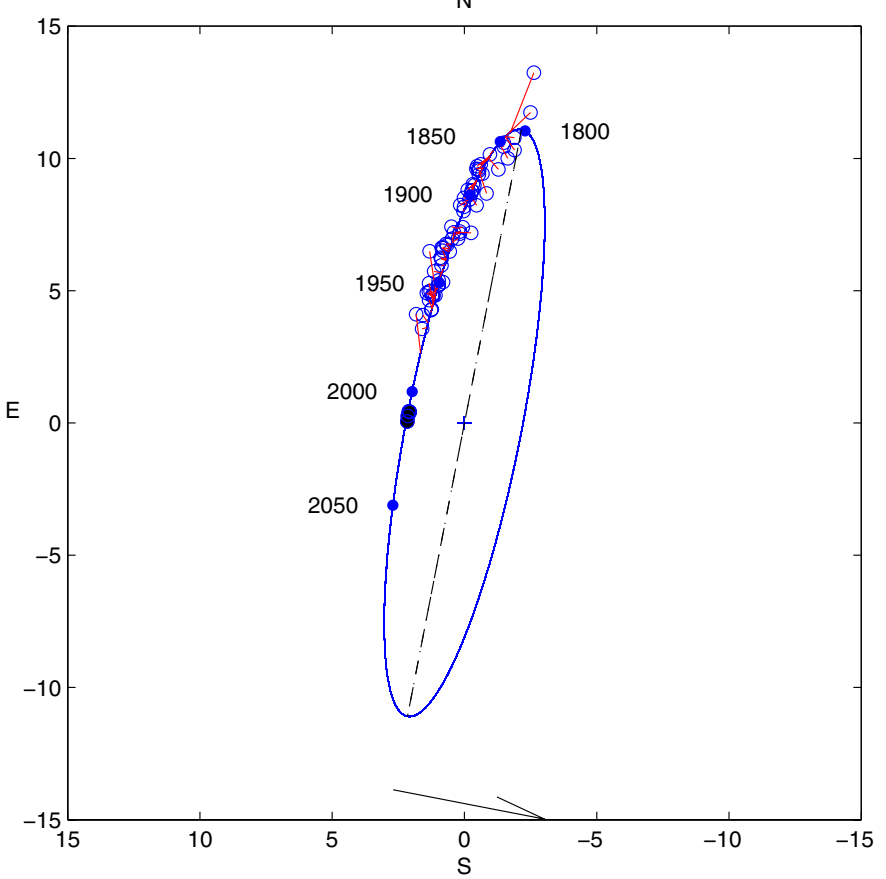

Figure 9. WDS 08592+4803 A-BC. Left: previous orbit of A around the barycenter of BC. Right: new orbit.

(A color version of this figure is available in the online journal.)

N

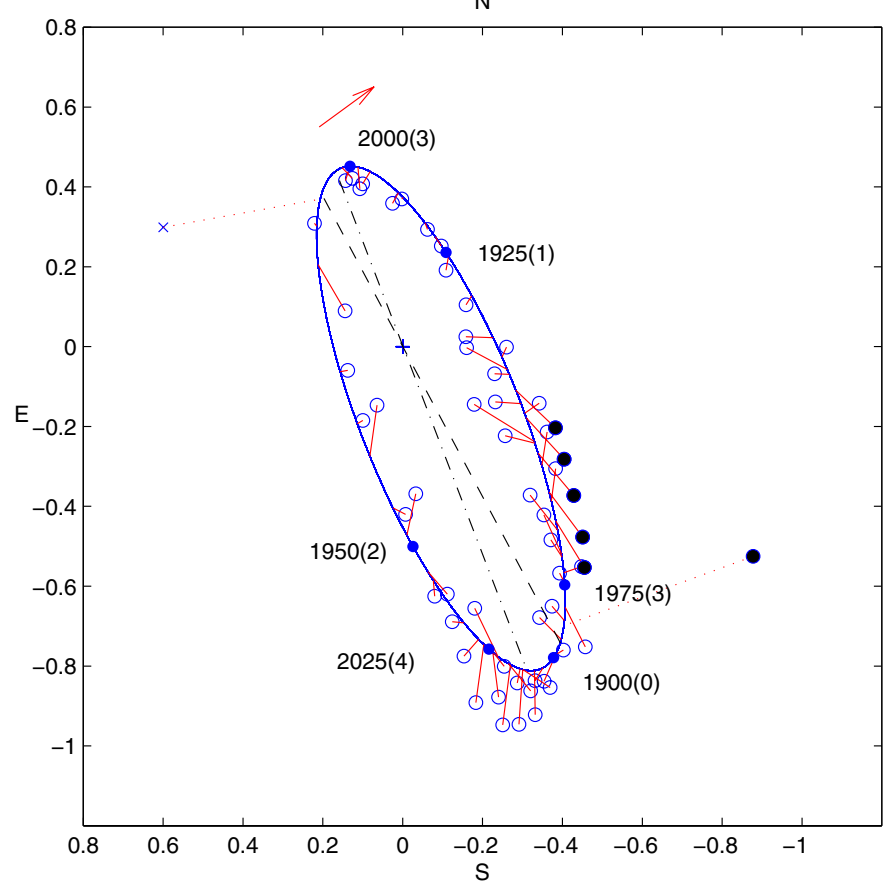

N

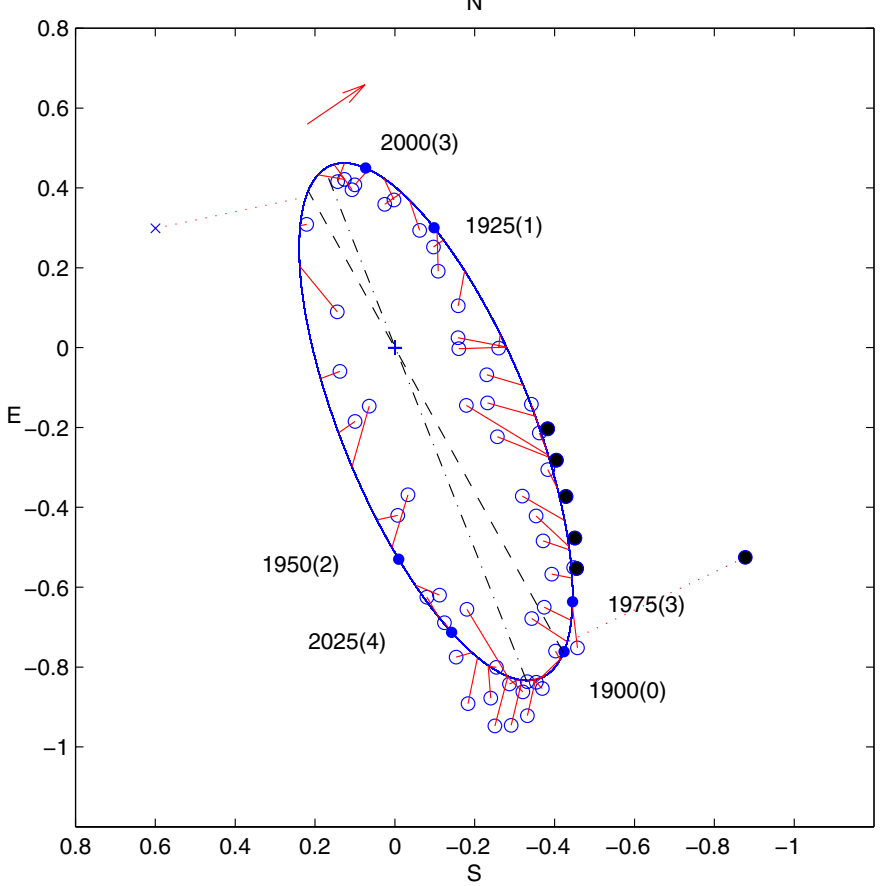

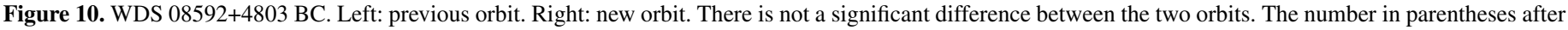
the year on the orbit indicates the lap number since first discovered in 1903. There may be evidence for sub-orbital motion indicating a third component.

(A color version of this figure is available in the online journal.) 

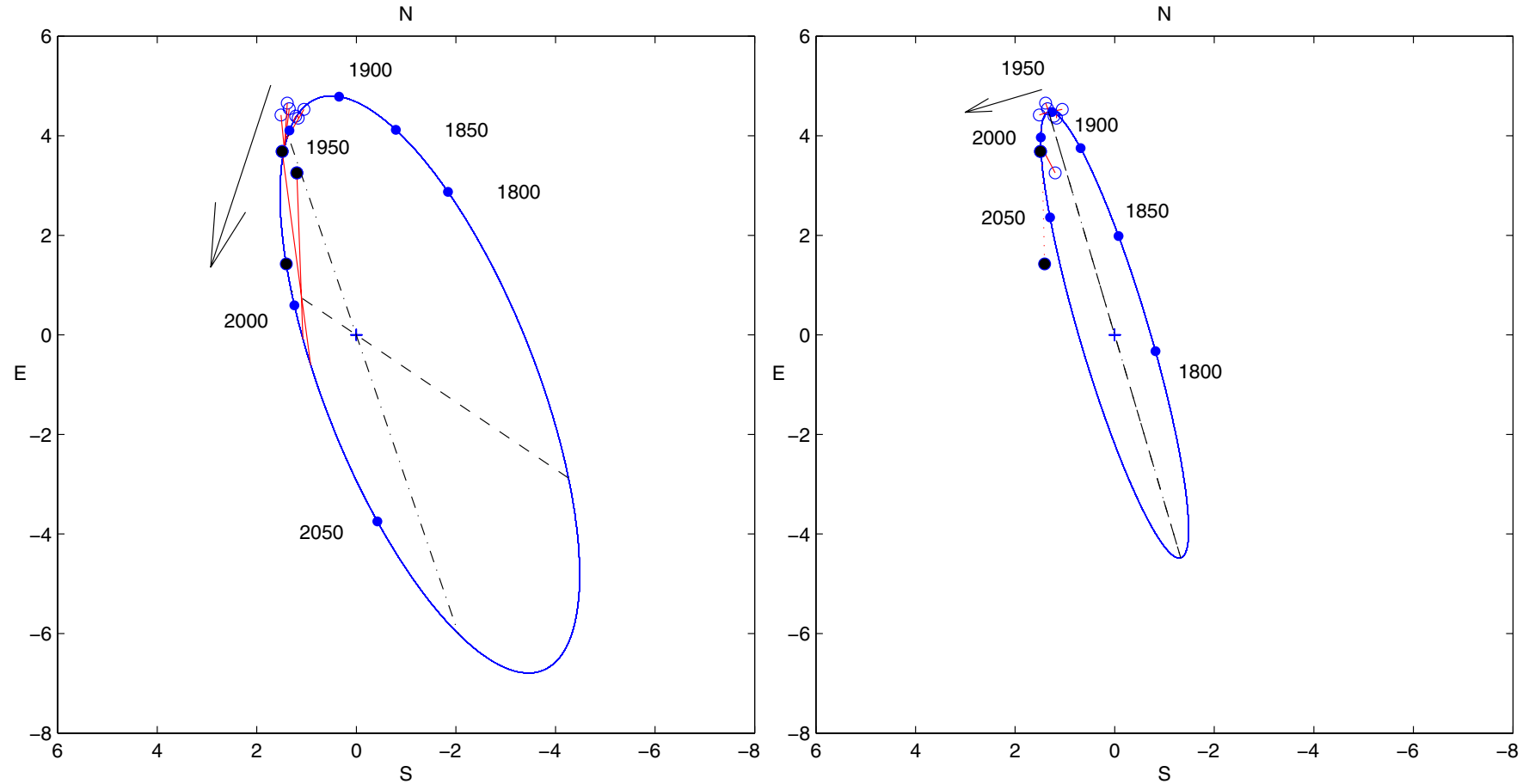

Figure 11. WDS 10281+4847. Left: previous orbit calculated before the last two measurements. Right: new orbit. Our measurement conflicts with the previous position, but both suggest that the antepenultimate measurement was in error. A new orbit is calculated, but both should be considered premature since so little of the orbital arc has been covered.

(A color version of this figure is available in the online journal.)
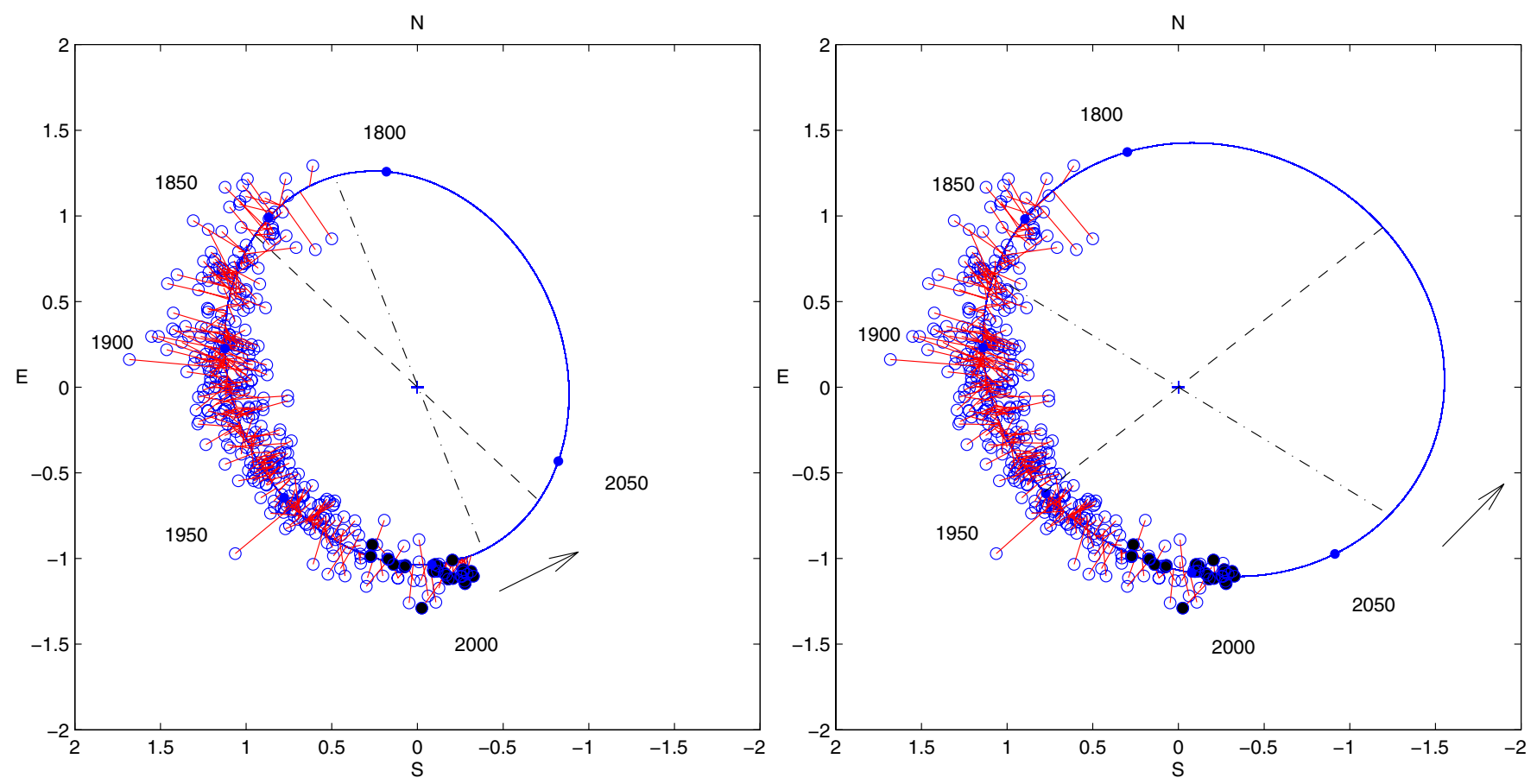

Figure 12. WDS 12533+2115. Left: previous orbit. Right: new orbit.

(A color version of this figure is available in the online journal.) 

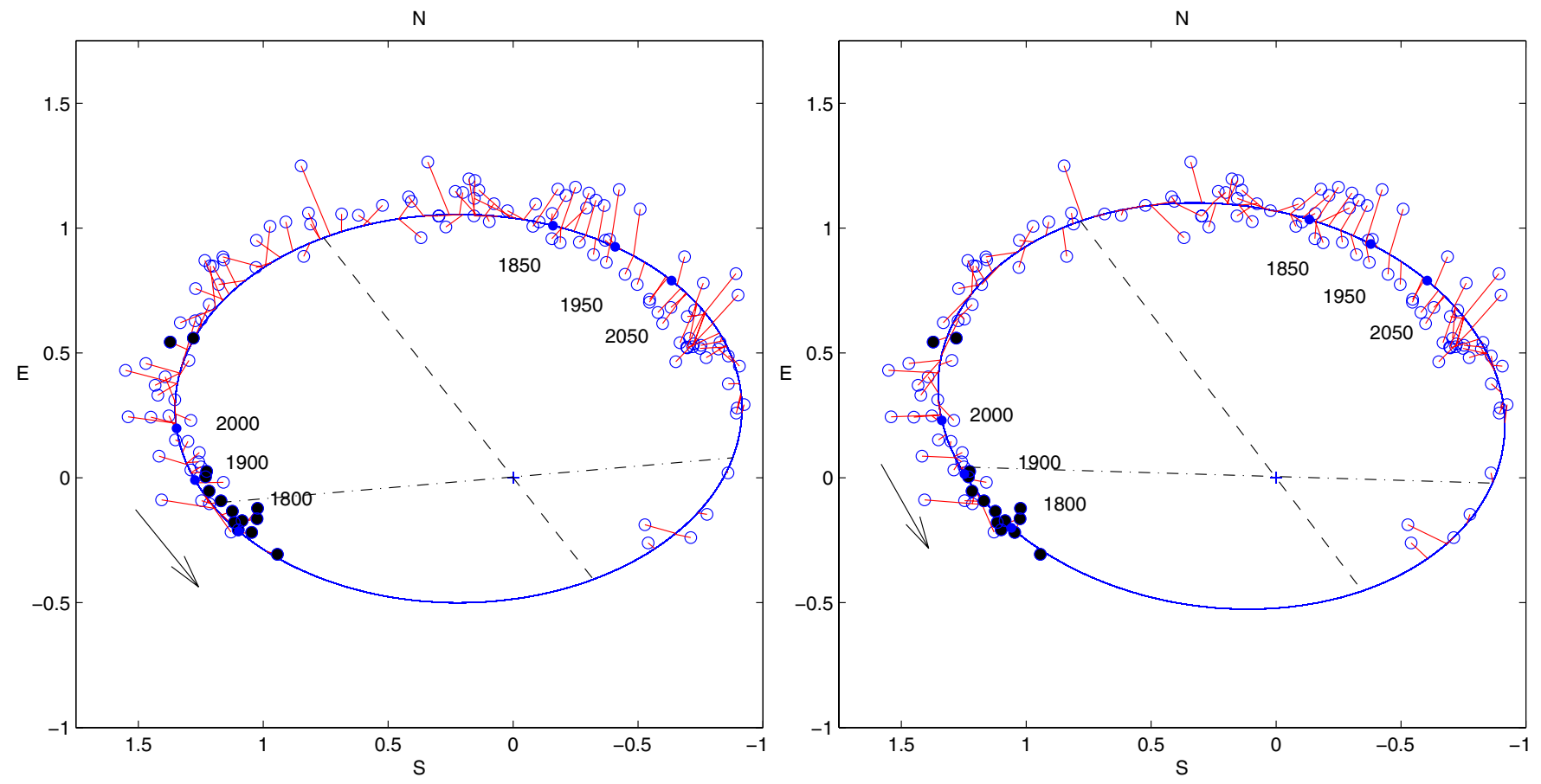

Figure 13. WDS $13007+5622$. Left: previous orbit. Right: new orbit. (A color version of this figure is available in the online journal.)
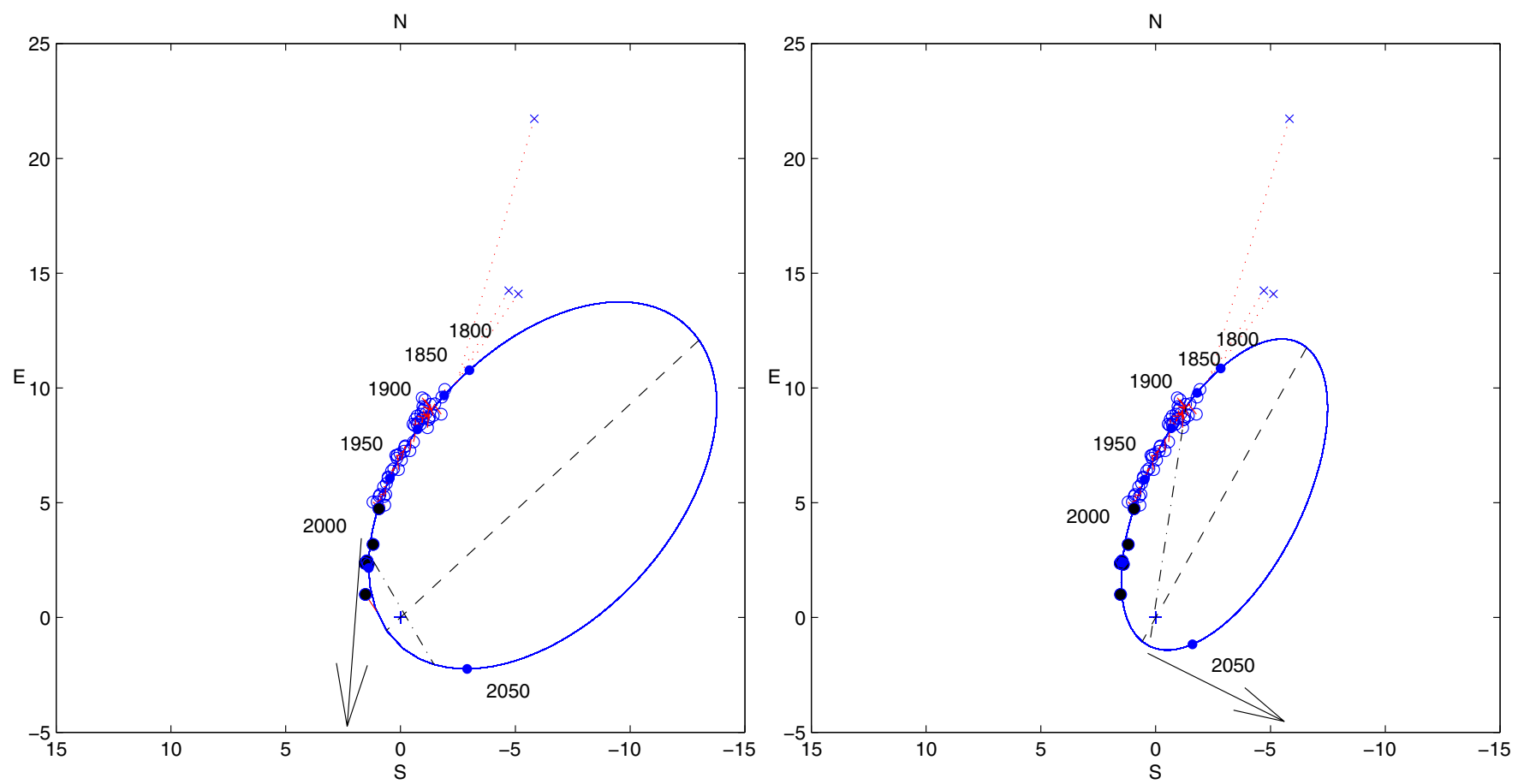

Figure 14. WDS 13473+1727. Left: previous orbit. Right: new orbit. (A color version of this figure is available in the online journal.) 

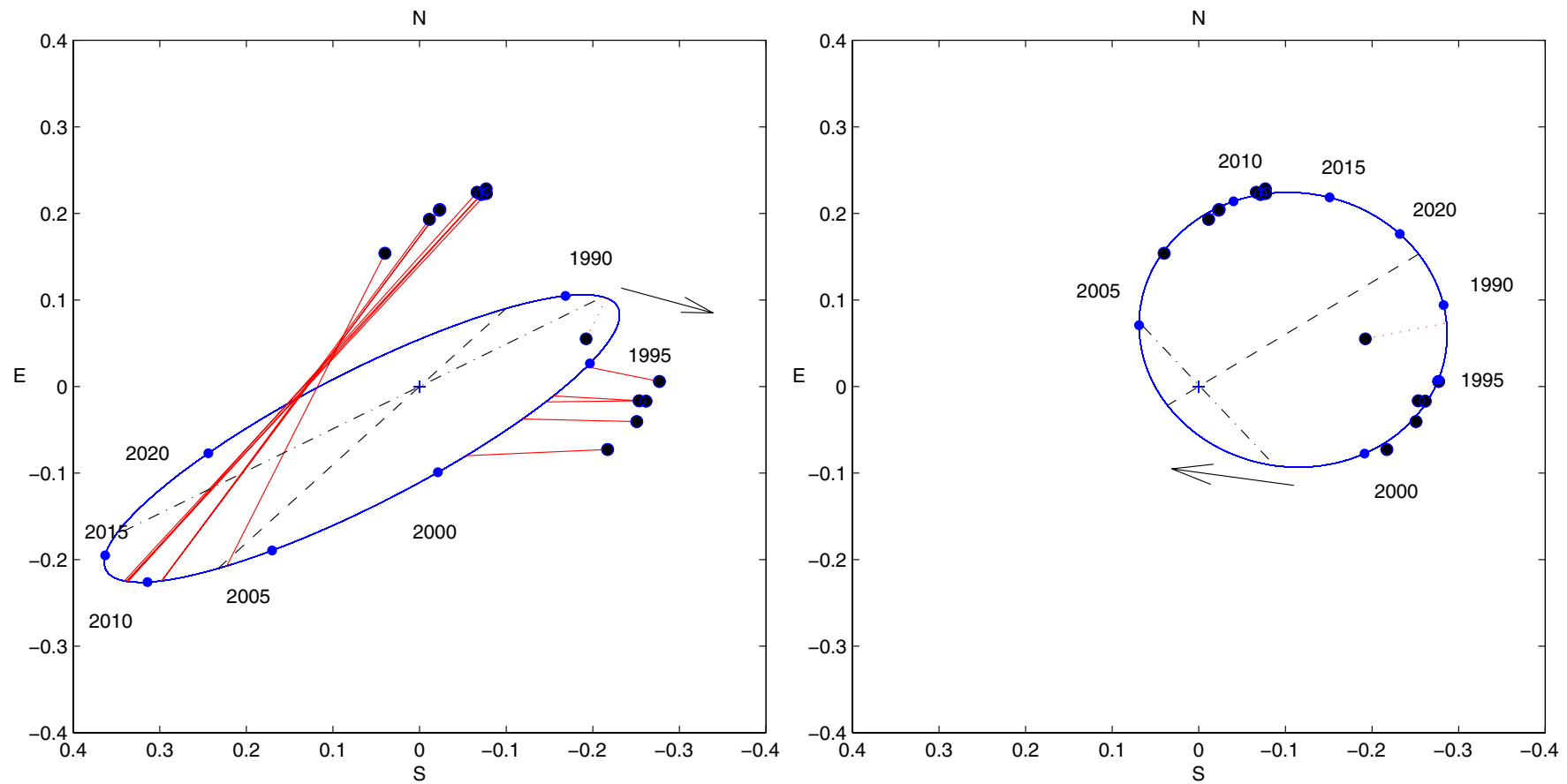

Figure 15. WDS 15496-0326. Left: previous orbit. Right: new orbit. $180^{\circ}$ have been added to all previously reported P.A.s in order to keep the brighter component as the center.

(A color version of this figure is available in the online journal.)

\section{CONCLUSIONS}

Finding that nearly one-fifth of 62 calibration binaries were discrepant emphasizes the need for including many binaries to calculate scale and orientation. On the other hand, it is reassuring that all of the discrepant binaries reported here could be reestablished as convenient calibration objects by merely updating their orbits. All 174 position measurements, not just the discrepant one, have been provided to the WDS.

The keepers of the WDS Library should be recognized for their important work: Brian D. Mason, Gary L. Wycoff, and William I. Hartkopf. Much more data and information is available at the WDS, and the keepers there are more than willing to provide assistance for interested users. For the current round, Brian Mason personally provided data for the binaries reported here. My thanks go out to the able observing crews at the SOR for obtaining measurements of the binaries, led by Test Directors Ryan Givens, Karl Schwenn, Jillian Conrad, Tod Laurvick, and Odell Reynolds.

\section{REFERENCES}

Baize, P. 1979, Inf. Circ., 79

Cvetkovic, Z. 2011, Inf. Circ., 174
De Rosa, R. J., Bulger, J., Patience, J., et al. 2011, MNRAS, 415, 854

Docobo, J. A., \& Ling, J. F. 1999, ApJS, 120, 41

Drummond, J. 1998, Proc. SPIE, 3353, 1050

Drummond, J. 2011, in Proceedings of the Advanced Maui Optical and Space Surveillance Technologies Conference, ed. S. Ryan (Kihei, HI: Maui Econ. Dev. Board), 500

Drummond, J. 2012, in Proceedings of the Advanced Maui Optical and Space Surveillance Technologies Conference, ed. S. Ryan (Kihei, HI: Maui Econ. Dev. Board), 535

Drummond, J., Fugate, R. Q., Christou, J. C., \& Hege, E. K. 1998, Icar, 132, 80 Eggen, O. J. 1967, ARA\&A, 5, 105

Gontcharov, G. A., \& Kiyaeva, O. V. 2010, NewA, 15, 324

Hale, A. 1994, AJ, 107, 306

Heintz, W. D. 1996, AJ, 111, 408

Heintz, W. D. 1997, ApJS, 111, 335

Hopmann, J. 1973, MiWie, 14, 18

Jancart, S., Jorissen, A., Babusiaux, C., \& Pourbaix, D. 2005, A\&A, 442, 365

Johnson, R., Montera, D., Schneeberger, T., \& Spinhirne, J. 2009, OSA Optics \& Photonics Technical Digest (CD), paper AOTuA1

Ling, J. F., Prieto, C., \& Magdalena, 2005, Inf. Circ., 155

Mason, B. D., McAllister, H. A., \& Hartkopf, W. L. 1995, AJ, 109, 332

Rica, F. W., Barrena, R., Vazquez, G., Henriquez, J. A., \& Hernandez, F. 2012, MNRAS, 419, 197

Roberts, L. C., Jr., Turner, N. H., ten Brummelaar, T. A., Mason, B. D., \& Hartkopf, W. I. 2011, AJ, 142, 175

Romero, F. M. 2013, Inf. Circ., 179

Scardia, M., Prieur, J.-L., Pansecchi, L., \& Argyle, R. W. 2012, Inf. Circ., 178 Scardia, M., Prieur, J.-L., Sala, M., et al. 2005, MNRAS, 357, 1255

van Leeuwen, F. 2007, A\&A, 474, 653 